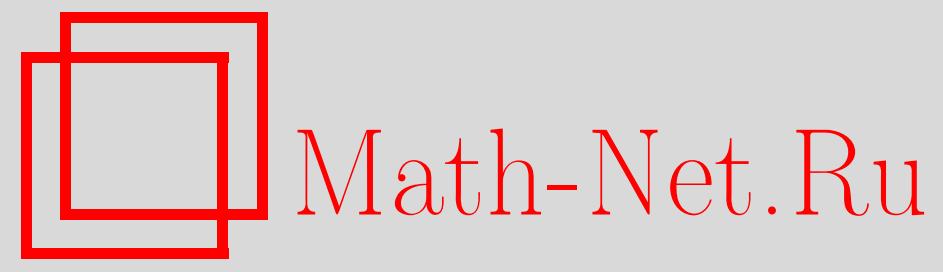

Е. Регаццини, В. В. Сазонов, Аппроксимация распределений случайных вероятностей смесями распределений Дирихле с приложениями к непараметрическим байесовским статистическим выводам, Теория вероятн. и ее примен., 2000, том 45, выпуск 1, 103-124

DOI: https://doi.org/10.4213/tvp326

Использование Общероссийского математического портала Math-Net.Ru подразумевает, что вы прочитали и согласны с пользовательским соглашением http://www . mathnet.ru/rus/agreement

Параметры загрузки:

IP : 35.174 .16 .151

26 апреля 2023 г., 15:58:24

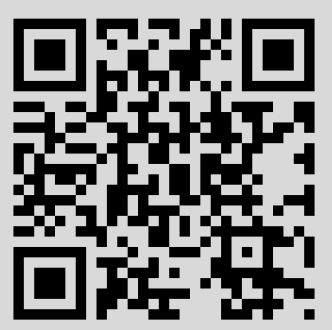




\title{
АППРОКСИМАЦИЯ РАСПРЕДЕЛЕНИЙ СЛУЧАЙНЫХ ВЕРОЯТНОСТЕЙ СМЕСЯМИ РАСПРЕДЕЛЕНИЙ ДИРИХЛЕ С ПРИЛОЖЕНИЯМИ К НЕПАРАМЕТРИЧЕСКИМ БАЙЕСОВСКИМ СТАТИСТИЧЕСКИМ ВЫВОДАМ ${ }^{1)}$
}

\begin{abstract}
В общей ситуации, характерной для непараметрического байесовского статистического анализа, когда наблюдения перестановочны и принимают значения в некотором польском пространстве $X$, априорные распределения аппроксимируются (в метрике Прохорова) с любой степенью точности явно конструируемыми смесями распределений процессов Дирихле. Показывается, что если такие смеси $\mathscr{P}_{n}$ слабо сходятся к некоторому заданному априорному распределению $\mathscr{P}$, то отвечающие $\mathscr{P}_{n}$ апостериорные распределения слабо сходятся к апостериорному распределению, отвечающему $\mathscr{P}$. При некоторых дополнительных ограничениях оценивается также точность аппроксимации. Эти результаты лежат в основе предлагаемого метода выбора априорного распределения, а также используются для аппроксимации предсказательного распределения (в вариационной метрике) и апостериорной функции распределения функционала $\int \psi d \widetilde{p}$ (в метрике Леви), где $\tilde{p}$ есть случайная вероятность с распределением $\mathscr{P}$.

Ключевые слова и фразы: аппрохсимация априорных и апостериорных распределений, распределения Дирихле, процессы Дирихле, выбор априорного распределения, метрика Леви, метрика Прохорова, случайные меры.
\end{abstract}

1. Введение. В статье изучается аппроксимация (в метрике Прохорова) априорного вероятностного распределения (в.р.) подходящей конечной смесью вероятностных мер (в.м.) Дирихле, определенных на множестве всех в.м., заданных на произвольном польском выборочном пространстве $X$. Кроме этого, для наблюдений, образующих перестановочную последовательность, оцениваются расстояния (также в метрике

\footnotetext{
${ }^{*}$ Dipartimento di Matematica, Università di Pavia, via Ferrata 1, 27100 Pavia, e CNRIAMI, Milano, Italia; e-mail: eugenio@dimat.unipv.it

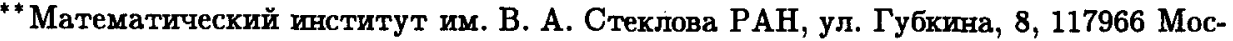
ква, ГСП-1, Россия; e-mail: sazonov@mi.ras.ru

1) Первый автор поддержан Progetto Strategico CNR (Decisioni statistiche: teoria e applicazioni) и Università «L. Bocconi» (Ricerca di base, 1997); второй автор поддержан the Cariplo Foundation for Scientific Research и проводил исследования во время пребыванпия в IMQ-Università «L. Bocconi», Milano.
} 
Регаччини Е., Сазонов В. В.

Прохорова) от апостериорного в.р. до апостериорных в.р., отвечающих указанным выше смесям в.м. Дирихле. Вероятностные меры Дирихле и их смеси весьма популярны в байесовской статистике. Одна из основных причин этого - тот факт, что апостериорное распределение, отвечающее априорному в.р. Дирихле (соответственно, являющемуся смесью в.р. Дирихле), само также есть в.р. Дирихле (соответственно, смесь в.р. Цирихле).

Материал статьи организован следующим образом. В п. 2 собраны некоторые полезные предварительные сведения. Отметим среди них определение и описание нескольких свойств гомеоморфизма, делающего строгим переход от априорного в.р., заданного на семействе всех в.м. на конечном множестве $X=\left\{a_{1}, \ldots, a_{k+1}\right\}$, к соответствуюшему априорному в.р. на $k$-мерном мультиномиальном параметре. В п. 3 мы определяем два типа дискретизации случайной вероятностной меры (с.в.м.) и оцениваем расстояние Прохорова между законом исходной с.в.м. и законами каждой из ее дискретизаций (предложение 1). Далее в п. 3 мы изучаем близость, в той же метрике, закона каждой из дискретизаций к подходящей смеси в.р. Дирихле. Результаты проведенного анализа суммируются в теореме 1. Теорема 2 в п. 4 представляет собой первую попытку количественного анализа близости апостериорных в.р., отвечающих некоторому априорному в.р., и смеси в.м. Дирихле, которая, по теореме 1, аппроксимирует исходное априорное вероятностное распределение. В действительности, однако, теорема 2 не содержит оценки близости апостериорных в.р. в какой-либо метрике, она утверждает лишь п.н. слабую сходимость, когда дискретизации становятся все более и более тонкими. Метрическая оценка близости апостериорных в.р. приводится в п. 5, где используется иная дискретизация, а также предполагаются выполненными некоторые дополнительные ограничения. Вторая часть п. 5 содержит ряд иллюстративных примеров. В заключительной части этого пункта рассматривағотя задачи аппроксимации предсказательного в.р. и апостериорного в.р. функционала $\int \psi d \widetilde{p}$, где $\widetilde{p}$ есть некоторая случайная вероятностная мера. В п. 6 обсуждаются возможные применения некоторых из результатов, полученных в предыдущих пунктах. В частности, показывается, что теорема 1 может быть использована для выбора априорного распределения.

Феномен близости двух апостериорных в.р., отвечающих двум близким априорным в.р., изучался также Краскером в [11] и Андреевым и Арьясом в [2]. Краскер рассматривал доминированное семейство вероятностей и, при выполнении некоторых довольно сильных ограничений на априорные в.р., показал, что если априорные в.р. близки в метрике Прохорова на «большом множестве», то их апостериорные распределения тоже близки в этой же метрике. Андреев и Арьяс рассматривали некоторое специальное доминированное семейство вероятностей - веро- 
ятности с непрерывными справа и имеющими конечные пределы слева плотностями по отношению к мере Лебега на $\mathbf{R}$, и, при выполнении ряда естественных условий, доказали, что если некоторая последовательность априорных в.р., сосредоточенных на ступенчатых функциях, слабо сходится к заданному в.р., то то же самое верно и для соответствующих апостериорных вероятностных распределений. Наши результаты по этой проблеме, содержашиеся в п. 4, имеют гораздо более широкую область применения.

2. Предварительные сведения. Всюду ниже $X$ обозначает некоторое польское пространство с метрикой $d_{X}$. Если $T-$ топологическое пространство, то $\mathscr{B}(T)$ будет обозначать его борелевскую $\sigma$-алгебру. Основным нашим вероятностным пространством будет $\left(X^{\infty}, \mathscr{B}\left(X^{\infty}\right), P\right)$, причем координатные случайные элементы $\left(\xi_{n}\right)_{n \geqslant 1}$ пространства $X^{\infty}$ предполагаются перестановочными (см., например, [1]) относительно $P$. Класс всех в.м. на $(X, \mathscr{B}(X))$, обозначаемый $M$, наделяется борелевской $\sigma$-алгеброй $\mathscr{B}(M)$, порожденной метрикой Прохорова $d$ (по поводу $d$ см. оригинальную работу Прохорова [12], либо [8]). Для любого натурального $n$

$$
\widetilde{e}_{n}(A):=\frac{1}{n} \sum_{i=1}^{n} \delta_{\xi_{i}}(A) \quad(A \in \mathscr{B}(X))
$$

есть пример с.в.м. на $(X, \mathscr{B}(X))$. Согласно общему определению, с.в.м. на $(X, \mathscr{B}(X))$ есть измеримое отображение пространства $\left(X^{\infty}, \mathscr{B}\left(X^{\infty}\right)\right)$ в $(M, \mathscr{B}(M))$.

Перечислим некоторые основные факты, касающиеся перестановочных последовательностей.

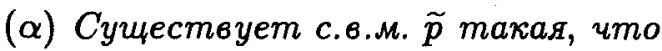

$$
\lim _{n \rightarrow+\infty} d\left(\tilde{e}_{n}, \tilde{p}\right)=0 \quad P-n . \mu .
$$

Справедлива теорема представления де Финетти, т.е. следующие утверждения экөивалентны:

( $\beta$ ) $\xi_{1}, \xi_{2}, \ldots$ перестановочны.

$(\gamma)$ Cуществует с.в.м. $\tilde{p}$ на $(X, \mathscr{B}(X))$ такая, что

$$
P(\xi \in B \mid \tilde{p})=\tilde{p}^{\infty}(B) \quad \text { P-n.н. }\left(B \in \mathscr{B}\left(X^{\infty}\right)\right)
$$

аде $\xi:=\left(\xi_{n}\right)_{n \geqslant 1}$ u $\tilde{p}^{\infty}$ есть счетное произведение $\tilde{p}$ на себя.

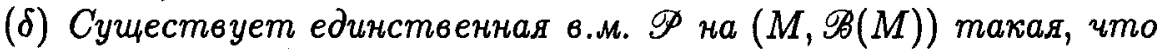

$$
P(\xi \in B)=\int_{M} p^{\infty}(B) \mathscr{P}(d p) \quad\left(B \in \mathscr{B}\left(X^{\infty}\right)\right)
$$


мера $\mathscr{P}$ называется мерой де Финетти последовательности $\xi:=$ $\left(\xi_{n}\right)_{n \geqslant 1}$ u coвпадает с в.p. случайной ө.м. $\tilde{p}$.

Если $\xi_{1}, \xi_{2}, \ldots$ перестановочны, то $\widetilde{p}$ в $(\alpha)$ и в $(\gamma)$ совпадают $P$-n.н.

Доказательства этих утверждений можно найти, например, в [1].

Мера де Финетти $\mathscr{P}$ в терминах байесовской статистики есть априорное в.р. в непараметрической ситуации.

Пусть $Y_{i}$ - независимые случайные величины с распределениями $\Gamma\left(\alpha_{i}, 1\right), \alpha_{i} \geqslant 0, i=1, \ldots, n, \alpha^{\prime}=\sum_{i=1}^{n} \alpha_{i}>0$ (когда $\alpha_{i}=0$, мы полагаем $\left.\Gamma\left(\alpha_{i}, 1\right)=\delta_{0}\right)$. Совместное распределение величин $Z_{i}=Y_{i} / \sum_{j=1}^{n} Y_{j}$, $i=1, \ldots, n-1$, обозначают $\mathscr{D}^{\left(\alpha^{\prime}\right)}\left(\cdot ; \alpha_{1}, \ldots, \alpha_{n-1}\right)$ и называют pacnpeделением Дирихле с параметром $\left(\alpha_{1}, \ldots, \alpha_{n}\right)$. Далее, пусть $\alpha$-мера на $(X, \mathscr{B}(X))$ такая, что $0<\alpha(X)<\infty$. С.в.м. называется прочессом Дирихле (см. [9]) или с.в.м. Дирихле с параметром $\alpha$, если для любого конечного измеримого разбиения $\left\{A_{1}, \ldots, A_{n}\right\}$ пространства $X$ вектор $\left(\tilde{p}\left(A_{1}\right), \ldots, \widetilde{p}\left(A_{n-1}\right)\right)$ имеет распределение $\mathscr{D}^{(\alpha(X))}\left(\cdot ; \alpha\left(A_{1}\right), \ldots, \alpha\left(A_{n-1}\right)\right)$. Распределение на $(M, \mathscr{B}(M))$, отвечающее процессу Дирихле с параметром $\alpha$, обозначим $\mathscr{D}(\cdot ; \alpha)$.

Пусть теперь $a_{i}$ - произвольные элементы пространства $X(i=$ $1, \ldots, k+1)$, п пусть $M_{1}$ обозначает замкнутое подмножество пространства $M$, состоящее из всех в.м. вида $\sum_{i=1}^{k+1} \theta_{i} \delta_{a_{i}}$, где $\theta=\left(\theta_{1}, \ldots, \theta_{k}\right) \in T_{k}$, $\theta_{k+1}=1-|\theta|,|\theta|=\sum_{i=1}^{k} \theta_{i}$ पI

$$
T_{k}=\left\{\left(\theta_{1}, \ldots, \theta_{k}\right) \in \mathbf{R}^{k}: \theta_{i} \geqslant 0 \quad(i=1, \ldots, k), \quad \sum_{i=1}^{k} \theta_{i} \leqslant 1\right\} .
$$

Рассмотрим функцию $t: M_{1} \rightarrow T_{k}$, отображающую $\sum_{i=1}^{k+1} \theta_{i} \delta_{a_{i}}$ в $\theta$. Покажем, что

$(\varepsilon) t: M_{1} \rightarrow T_{k}$ есть гомеоморфизм $u$, более того,

$$
\frac{1}{2}\left\|\theta-\theta^{\prime}\right\| \leqslant d\left(\bar{\theta}, \bar{\theta}^{\prime}\right) \leqslant \sqrt{k}\left\|\theta-\theta^{\prime}\right\|
$$

для любых $\theta, \theta^{\prime}$ из $T_{k}$, где $\|\cdot\|$ есть евклидова норма в $\mathbf{R}^{k}, d$ есть метрика Прохорова в $M u \bar{\theta}=\sum_{i=1}^{k+1} \theta_{i} \delta_{a_{i}}=t^{-1}(\theta), \bar{\theta}^{\prime}=\sum_{i=1}^{k+1} \theta_{i}^{\prime} \delta_{a_{i}}=t^{-1}\left(\theta^{\prime}\right)$.

Для доказательства этого утверждения достаточно, конечно, доказать справедливость неравенств между метриками. С этой целью заметим, что для любого $E \subset X$

$$
\sum_{i=1}^{k+1} \theta_{i} \delta_{a_{i}}(E) \leqslant \sum_{i=1}^{k+1} \theta_{i}^{\prime} \delta_{a_{i}}(E)+\sum_{i=1}^{k+1}\left(\theta_{i}-\theta_{i}^{\prime}\right)^{+} .
$$

Отсюда легко следует, что

$$
d\left(\bar{\theta}, \bar{\theta}^{\prime}\right)=\sum_{i=1}^{k+1}\left(\theta_{i}-\theta_{i}^{\prime}\right)^{+}=\frac{1}{2} \sum_{i=1}^{k+1}\left|\theta_{i}-\theta_{i}^{\prime}\right| .
$$


Следовательно,

$$
\left\|\theta-\theta^{\prime}\right\| \leqslant \sum_{i=1}^{k}\left|\theta_{i}-\theta_{i}^{\prime}\right| \leqslant 2 d\left(\bar{\theta}, \bar{\theta}^{\prime}\right) .
$$

С другой стороны, поскольку $2 d\left(\bar{\theta}, \bar{\theta}^{\prime}\right)=\sum_{i=1}^{k}\left|\theta_{i}-\theta_{i}^{\prime}\right|+\left|\sum_{i=1}^{k}\left(\theta_{i}-\theta_{i}^{\prime}\right)\right|$, то

$$
d\left(\bar{\theta}, \bar{\theta}^{\prime}\right) \leqslant \sum_{i=1}^{k}\left|\theta_{i}-\theta_{i}^{\prime}\right| \leqslant \sqrt{k}\left\|\theta-\theta^{\prime}\right\| .
$$

Ниже нам потребуется следующий факт, непосредственно вытекающий из $(\varepsilon)$.

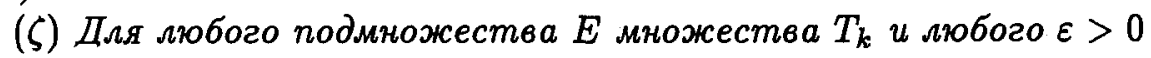

$$
E^{\varepsilon]} \subset t\left(\left(t^{-1}(E)\right)^{\varepsilon \sqrt{k}]}\right) \quad u \quad t\left(\left(t^{-1}(E)\right)^{\epsilon}\right) \subset E^{2 \epsilon}
$$

где $A^{\varepsilon]}$ обозначает нножество всех элементов с расстоянием до $A$, не превосходящим $\varepsilon$.

Определим $\mu^{(n)}$, положив

$$
\mu^{(n)}(A)=\mathbf{E}\left(\tilde{p}^{n}(A)\right) \quad(A \in \mathscr{B}\left(X^{n}\right), \widetilde{p}^{n}=\underbrace{\widetilde{p} \times \cdots \times \tilde{p}}_{n}),
$$

и пусть $x^{(n)} \mapsto \mathscr{P}\left(\cdot ; x^{(n)}\right), x^{(n)} \in X^{n}$, есть регулярное условное в.р. $\tilde{p}$ при условии $\left\{\xi^{(n)}=x^{(n)}\right\}$, где $\xi^{(n)}=\left(\xi_{1}, \ldots, \xi_{n}\right)$.

Наконец, сделаем еще одно замечание, которое будет полезным впоследствии. Предположим, что значения $\tilde{p}$ принадлежат $M_{1}$. Тогда $\tilde{p}=\sum_{i=1}^{k+1} \tilde{p}\left(a_{i}\right) \delta_{a_{i}}$ и для ее распределения $\mathscr{P}$ на $(M, \mathscr{B}(M))$ мы имеем

$$
\begin{aligned}
\mathscr{P}(E) & =\mathscr{P}\left(E \cap M_{1}\right)=P\left(\tilde{p} \in E \cap M_{1}\right) \\
& =P\left(\left(\tilde{p}\left(a_{1}\right), \ldots, \tilde{p}\left(a_{k}\right)\right) \in t\left(E \cap M_{1}\right)\right)=P_{F}\left(t\left(E \cap M_{1}\right)\right)
\end{aligned}
$$

где $P_{F}$ есть распределение случайного вектора $\left(\widetilde{p}\left(a_{1}\right), \ldots, \widetilde{p}\left(a_{k}\right)\right)$.

3. Аппроксимация априорных вероятностных распределений смесями вероятностных распределений Дирихле. Поскольку пространство $X$ сепарабельно, для любого $\varepsilon>0$ существует его вполне ограниченное подмножество $K_{\varepsilon}$ такое, что $\mu\left(K_{\varepsilon}^{c}\right) \leqslant \varepsilon^{2}$, где для простоты мы пишем $\mu$ вместо $\mu^{(1)}$ (см., например, доказательство теоремы 1.4 в [3]). Пусть $\left\{A_{1}, \ldots, A_{k}\right\}$ есть измеримое разбиение $K_{\varepsilon}$ такое, что $\varepsilon \geqslant \operatorname{diam}\left(A_{i}\right):=\sup _{x, y \in A_{i}} d_{X}(x, y)$ при всех $i=1, \ldots, k$. Случайную в.м.

$$
\tilde{p}_{\varepsilon}=\sum_{i=1}^{k+1} \tilde{p}\left(A_{i}\right) \delta_{a_{i}}
$$


где $A_{k+1}:=K_{\varepsilon}^{c}$ и $a_{i}$ есть фиксированная точка из $A_{i}(i=1, \ldots, k+1)$, назовем $(\varepsilon, \mu)$-дискретизачией $\widetilde{p}$.

Чтобы избежать использования $\mu$ при построении разбиения, входяшего в определение $\widetilde{p}_{\varepsilon}$, можно использовать тот факт, что сепарабельность влечет существование эквивалентной вполне ограниченной метрики $d_{X}^{\prime}$ в $X$. Если $X$ рассматривать с метрикой $d_{X}^{\prime}$, то для любого $\varepsilon>0$ существует конечное измеримое разбиение - для простоты мы обозначим его по-прежнему $\left\{A_{1}, \ldots, A_{k+1}\right\}-$ такое, что $\operatorname{diam}\left(A_{i}\right) \leqslant \varepsilon$ $(i=1, \ldots, k+1)$. Отвечающее этому разбиению $\widetilde{p}_{\varepsilon}$ назовем $\varepsilon$-вполне ограниченной дискретизачией $\widetilde{p}$. Когда рассматривается $\varepsilon$-вполне ограниченная дискретизация, метрика Прохорова в $M$ определяется относительно $d_{X}^{\prime}$. Следует отметить, что использование метрики $d_{X}^{\prime}$ вместо $d_{X}$ может исказить картину наблюдений, например, когда представляют интерес попарные расстояния между наблюдениями.

Всюду в дальнейшем $\mathscr{P}_{\varepsilon}$ и $F_{\varepsilon}$ обозначают, соответственно, в.р. случайной в.м. $\widetilde{p}_{\varepsilon}$ и функцию распределения (Ф.р.) случайного вектора $\left(\tilde{p}\left(A_{1}\right), \ldots, \tilde{p}\left(A_{k}\right)\right)$. В следующем предложении оценивается близость $\mathscr{P}_{\varepsilon}$ и $\mathscr{P}$.

Предложение 1. Если $\tilde{p}_{\varepsilon}$ есть $(\varepsilon, \mu)$ - или $\varepsilon$-вполне ограниченная дискретизачия $\tilde{p}, \bmod \left(\mathscr{P}_{\varepsilon}, \mathscr{P}\right) \leqslant \varepsilon$.

Д о к а з а т е л ь с т в о. Если $A, B$ суть замкнутые подмножества $M$ и $X$ соответственно, а $\widetilde{p}_{\varepsilon}$ есть вполне ограниченная дискретизация, то

$$
\begin{aligned}
\tilde{p}_{\varepsilon}(B) & =\sum_{i=1}^{k+1} \tilde{p}\left(A_{i}\right) \delta_{a_{i}}(B)=\sum_{\left\{i: a_{i} \in B\right\}} \tilde{p}\left(A_{i}\right) \\
& \leqslant \tilde{p}\left(B^{\epsilon]}\right) \quad\left[\text { поскольку } \operatorname{diam}\left(A_{i}\right) \leqslant \varepsilon, \forall i\right],
\end{aligned}
$$

и поэтому $d\left(\widetilde{p}_{\varepsilon}, \tilde{p}\right) \leqslant \varepsilon$. Следовательно, если $\widetilde{p} \in A$, то $\widetilde{p}_{\varepsilon} \in A^{\varepsilon]}$ и

$$
\mathscr{P}(A)=P(\{\tilde{p} \in A\}) \leqslant P\left(\left\{\widetilde{p}_{\varepsilon} \in A^{\varepsilon]}\right\}\right)=\mathscr{P}_{\varepsilon}\left(A^{\varepsilon]}\right),
$$

что влечет $d\left(\mathscr{P}_{\varepsilon}, \mathscr{P}\right) \leqslant \varepsilon$.

Если $\tilde{p}_{\varepsilon}$ есть $(\varepsilon, \mu)$-дискретизация $\widetilde{p}$, то положим

$$
\Omega_{\varepsilon}=\left\{x \in X^{\infty}: \widetilde{p}\left(A_{k+1}\right) \leqslant \varepsilon\right\}
$$

и заметим, что по неравенству Маркова

$$
\mathscr{P}(A) \leqslant P\left(\{\tilde{p} \in A\} \cap \Omega_{\varepsilon}\right)+\varepsilon .
$$

Поскольку при $x \in \Omega_{\varepsilon}$

$$
\tilde{p}_{\varepsilon}(B) \leqslant \sum_{\left\{i \leqslant k: a_{i} \in B\right\}} \tilde{p}\left(A_{i}\right)+\varepsilon \leqslant \tilde{p}\left(B^{\varepsilon]}\right)+\varepsilon
$$


для таких $x$ мы имеем $d\left(\tilde{p}_{\varepsilon}, \tilde{p}\right) \leqslant \varepsilon$, так что

$$
P\left(\{\widetilde{p} \in A\} \cap \Omega_{\varepsilon}\right) \leqslant P\left(\tilde{p}_{\varepsilon} \in A^{\varepsilon]}\right)=\mathscr{P}_{\varepsilon}\left(A^{\varepsilon]}\right) .
$$

\section{Предложение 1 доказано.}

Пусть $M_{\varepsilon}$ есть класс всех $p \in M$ с носителем, содержащимся в множестве точек $\left\{a_{1}, \ldots, a_{k+1}\right\}$, использованном при определении дискретизаций, и пусть $t: M_{\varepsilon} \rightarrow T_{k}$ есть гомеоморфизм, определенный как в предыдущем пункте. Тогда, как было отмечено в конце предыдущего пункта,

$$
\mathscr{P}_{\varepsilon}(E)=\mathscr{P}_{\varepsilon}\left(E \cap M_{\varepsilon}\right)=P_{F_{\varepsilon}}\left(t\left(E \cap M_{\varepsilon}\right)\right) \quad(E \in \mathscr{B}(M))
$$

Как показано в разделе 4 нашей работы $[13], P_{F_{c}}$ может быть приближена смесью

$$
\varphi_{\lambda, \mu}^{(\varepsilon)}(B)=\sum_{\nu} P_{F_{\varepsilon}}\left(\Delta_{\nu}\right) \mathscr{D}^{(\lambda)}(B ; \mu(\nu)) \quad\left(B \in \mathscr{B}\left([0,1]^{k}\right)\right)
$$

где $\mu(\nu)=\left(\mu_{1}, \ldots, \mu_{k}\right), \mu_{i}=\lambda \tau_{i}(i=1, \ldots, k)$,

$$
\tau_{i}=\left\{\begin{array}{ll}
0, & \text { если } \nu_{i}=0, \\
\frac{\nu_{i}-b_{k}}{N}, & \text { если } \nu_{i} \geqslant 1,
\end{array} \quad i=1, \ldots, k,\right.
$$

$\nu \in I:=\left\{\left(\nu_{1}, \ldots, \nu_{k}\right) \in\{0,1, \ldots, N\}^{k}:|\nu| \leqslant N+k-1\right\}, \Delta_{\nu}=\times_{i=1}^{k}\left(\left(\nu_{i}-\right.\right.$ 1) $\left./ N, \nu_{i} / N\right], b_{k}$ - рациональное число из интервала $((k-1) / k, 1)$ и $\lambda$ натуральное число такое, что

$$
\lambda \geqslant 1+\frac{N^{2}}{2 g_{k}^{2}} \ln \frac{2 k}{\varepsilon}, \quad \mu_{i} \in \mathbf{N} \quad\left(\nu \in I \mathrm{c} \nu_{i} \geqslant 1, i=1, \ldots, k\right),
$$

где $g_{k}=b_{k} \wedge\left(1-b_{k}\right)$. Определим теперь

$$
\mathscr{P}_{\lambda, \mu}^{(\varepsilon)}(E):=\sum_{\nu} P_{F_{\varepsilon}}\left(\Delta_{\nu}\right) \mathscr{D}\left(E ; \alpha_{\mu(\nu)}\right) \quad(E \in \mathscr{B}(M))
$$

с $\nu, \lambda, \mu$ такими же, как выше, и $\alpha_{\mu(\nu)}:=\sum_{i=1}^{k+1} \mu_{i} \delta_{a_{i}}$, и отметим, что

$$
\mathscr{P}_{\lambda, \mu}^{(\varepsilon)}(E)=\varphi_{\lambda, \mu}^{(\varepsilon)}\left(t\left(E \cap M_{\varepsilon}\right)\right) \quad(E \in \mathscr{B}(M))
$$

Действительно,

$$
\begin{aligned}
& \sum_{\nu} P_{F_{\varepsilon}}\left(\Delta_{\nu}\right) \mathscr{D}\left(E ; \alpha_{\mu(\nu)}\right) \\
& =\sum_{\nu} P_{F_{\varepsilon}}\left(\Delta_{\nu}\right) \mathscr{D}\left(E \cap M_{\varepsilon} ; \alpha_{\mu(\nu)}\right) \\
& \quad\left[\text { поскольку носитель } \mathscr{D}\left(\cdot ; \alpha_{\mu(\nu)}\right) \text { принадлежит } M_{\varepsilon}\right] \\
& =\varphi_{\lambda, \mu}^{(\varepsilon)}\left(t\left(E \cap M_{\varepsilon}\right)\right) .
\end{aligned}
$$


Установив связь между $\mathscr{P}_{\lambda, \mu}^{(\varepsilon)}$ и $\varphi_{\lambda, \mu}^{(\varepsilon)}$, мы теперь в состоянии доказать следуюшую теорему.

Теорема 1. Если $\tilde{p}_{\varepsilon}$ есть либо $(\varepsilon, \mu)-$, либо $\varepsilon$-вполне ограниченнал дискретизачия $\tilde{p}$ и если в.м. $\mathscr{P}_{\lambda, \mu}^{(\varepsilon)}$ определена равенством (3.3) с $N>$ $k / \varepsilon$ u $\lambda$, удовлетөоряюиим $(3.2), \bmod \left(\mathscr{P}, \mathscr{P}_{\lambda, \mu}^{(\varepsilon)}\right) \leqslant 2 \varepsilon$.

Д о к а з т е л в с т в о. Для любого замкнутого $E \subset M$

$$
\begin{aligned}
\mathscr{P}_{\lambda, \mu}^{(\varepsilon)}(E)= & \mathscr{P}_{\lambda, \mu}^{(\varepsilon)}\left(E \cap M_{\varepsilon}\right)=\varphi_{\lambda, \mu}^{(\varepsilon)}\left(t\left(E \cap M_{\varepsilon}\right)\right) \\
\leqslant & P_{F_{\varepsilon}}\left(\left(t\left(E \cap M_{\varepsilon}\right)\right)^{\sqrt{k} / N}\right)+\varepsilon \\
& \quad[\text { ср. доказательство теоремы } 1 \text { в }[13]] \\
= & \mathscr{P}_{\varepsilon}\left(t^{-1}\left(\left(t\left(E \cap M_{\varepsilon}\right)\right)^{\sqrt{k} / N}\right)\right)+\varepsilon \\
\leqslant & \mathscr{P}_{\varepsilon}\left(\left(E \cap M_{\varepsilon}\right)^{k / N]}\right)+\varepsilon \quad[\text { в силу }(\zeta)] .
\end{aligned}
$$

Следовательно, $d\left(\mathscr{P}_{\varepsilon}, \mathscr{P}_{\lambda, \mu}^{(\varepsilon)}\right) \leqslant \varepsilon$ при $N>k / \varepsilon$ и

$$
\begin{aligned}
d\left(\mathscr{P}, \mathscr{P}_{\lambda, \mu}^{(\varepsilon)}\right) & \leqslant d\left(\mathscr{P}, \mathscr{P}_{\varepsilon}\right)+d\left(\mathscr{P}_{\varepsilon}, \mathscr{P}_{\lambda, \mu}^{(\varepsilon)}\right) \\
& \leqslant 2 \varepsilon \quad \text { [по предложению 1]. }
\end{aligned}
$$

Теорема 1 доказана.

4. Aпmроксимация апостериорного вероятностного распределения: общий случай. В настоящем пункте предполагается, что пространство $X$ вполне ограничено. Рассмотрим последовательность конечных измеримых разбиений $X$

$$
\Pi_{m}=\left\{A_{m, 1}, \ldots, A_{m, k_{m}+1}\right\} \quad(m=1,2, \ldots)
$$

и положим

$$
\varepsilon_{m}=\max _{1 \leqslant i \leqslant k_{m}+1} \operatorname{diam}\left(A_{m, i}\right) .
$$

Введем следующие условия:

$$
\begin{array}{ll}
\Pi_{m+1} & \text { есть измельчение } \Pi_{m} \text { для всех } m ; \\
\mathscr{B}(X) & \text { порождается классом множеств } \cup_{m} \Pi_{m} ; \\
\varepsilon_{m} \downarrow 0 & \text { при } m \rightarrow+\infty .
\end{array}
$$

Ясно, что при всех $p$ из $M$ и $m$ из $\mathbf{N}$

$$
p_{\varepsilon_{m}}:=\sum_{i=1}^{k_{m}+1} p\left(A_{m, i}\right) \delta_{a_{m, i}} \quad\left(a_{m, i} \in A_{m, i}: i=1, \ldots, k_{m}+1\right)
$$


есть $\varepsilon_{m}$-вполне ограниченная дискретизация $p$. Для любых фиксированных $n$ и $m$ из $\mathbf{N}$ определим случайные элементы $\xi_{r, m}(1 \leqslant r \leqslant n)$ на $X^{\infty}$, положив

$$
\xi_{r, m}(x)=a_{m, i}, \quad \text { если } x_{r} \in A_{m, i}\left(i=1, \ldots, k_{m}+1 ; r=1, \ldots, n\right),
$$

и для каждого $j=\left(j_{1}, \ldots, j_{n}\right)$ из $\left\{1, \ldots, k_{m}+1\right\}^{n}$ обозначим $R_{m, j}$ прямоугольник $A_{m, j_{1}} \times \cdots \times A_{m, j_{n}}$. Эти прямоугольники образуют разбиение $X^{n}$, которое порождает $\sigma$-алгебру $\mathscr{X}_{m}^{n}$. Положим теперь $\xi_{m}^{(n)}=$ $\left(\xi_{1, m}, \ldots, \xi_{n, m}\right)$ и рассмотрим регулярное условное в.p. $\xi$ при заданном $\xi_{m}^{(n)}$,

$$
x \mapsto P\left(\cdot \mid \xi_{m}^{(n)}\right)(x) \quad\left(x \in X^{\infty}\right),
$$

считая при этом, не теряя существенной общности, что $\mu^{(n)}\left(R_{m, j}\right)>0$ при всех $j$. Имеем п.н.

$$
\begin{aligned}
& P\left(\tilde{p} \in A \mid \xi_{m}^{(n)}\right)(x) \\
& \quad=\sum_{j} \mathbf{1}_{R_{m, j}}\left(x^{(n)}\right) \frac{\int_{A} p^{n}\left(R_{m, j}\right) \mathscr{P}(d p)}{\mu^{(n)}\left(R_{m, j}\right)} \quad\left(A \in \mathscr{B}(M), x \in X^{\infty}\right),
\end{aligned}
$$

где, как обычно, $x^{(n)}$ при $x=\left(x_{1}, x_{2}, \ldots\right)$ означает $\left(x_{1}, \ldots, x_{n}\right)$. Отметим, что это условное в.р. для каждого $x$ зависит лишь от $x^{(n)}$, и покажем, что при выполнении (4.1)-(4.2) оно сходится к $\mathscr{P}\left(\cdot ; x^{(n)}\right)$ п.н.

Предложение 2. При выполнении условии (4.1)-(4.2) существует множество $\mathcal{N}$ из $\mathscr{B}\left(X^{n}\right)$ maкое, ито $\mu^{(n)}(\mathscr{N})=0 u$

$$
d\left(P\left(\tilde{p} \in \cdot \mid \xi_{m}^{(n)}\right)(x), \mathscr{P}\left(\cdot ; x^{(n)}\right)\right) \longrightarrow 0 \quad n p u m \rightarrow+\infty
$$

для всех $x$ из $X^{\infty}$ с $x^{(n)} \in \mathcal{N}^{c}$. (Отметим, что разбиения, удовлетворяюшие (4.1)-(4.2), существуют в любом сепарабельном метрическом пространстве.)

Д ок а з а т е л ь с т в о. Поскольку $\xi_{m}^{(n)}$ порождает $\sigma$-алгебру $\{A \times$ $\left.X^{\infty}: A \in \mathscr{X}_{m}^{n}\right\}, \mathscr{X}_{m}^{n} \subset \mathscr{X}_{m+1}^{n}(m=1,2, \ldots)$ и $\bigcup_{m} \mathscr{X}_{m}^{n}$ порождает $\mathscr{B}\left(X^{n}\right)$, имеем

$$
P\left(\tilde{p} \in A \mid \xi_{m}^{(n)}\right)(x) \rightarrow \mathscr{P}\left(A ; x^{(n)}\right) \quad(m \rightarrow+\infty)
$$

для всех $x$ таких, что $x^{(n)} \in \mathscr{N}_{A}^{c}$, где множество $\mathscr{N}_{A}$ таково, что $\mu^{(n)}\left(\mathcal{N}_{A}\right)=0$ (см., например, теорему 35.6 в [4]). Рассмотрим теперь класс $\mathscr{I}$ всех конечных пересечений шаров в $M$ с рациональными радиусами и центрами в точках некоторого счетного плотного подмножества $M$. Ясно, что $\mu^{(n)}\left(\cup_{A \in \mathscr{I}} \mathscr{N}_{A}\right)=0$ и для любого $x$ с $x^{(n)} \in\left(\cup_{A \in \mathscr{J}} \mathcal{N}_{A}\right)^{c}$ и любого $A$ из $\mathscr{I}$, согласно доказанному выше, имеем $P\left(\tilde{p} \in A \mid \xi_{m}^{(n)}\right)(x) \longrightarrow \mathscr{P}\left(A ; x^{(n)}\right)$ при $m \rightarrow+\infty$. Утверждение предложения вытекает теперь из следствия 1 теоремы 2.2 в [3], если положить $\mathscr{N}=\cup_{A \in \mathcal{I}} \mathscr{N}_{A}$. 
Предложение 2 утверждает, что $\mathscr{P}\left(\cdot ; \boldsymbol{x}^{(n)}\right)$ можно аппроксимировать посредством $P\left(\tilde{p} \in \cdot \mid \xi_{m}^{(n)}\right)(x)$ для почти всех $x^{(n)}$. В свою очередь, последнее выражение можно аппроксимировать посредством

$P\left(\widetilde{p}_{\varepsilon_{m}} \in A \mid \xi_{m}^{(n)}\right)(x)=\sum_{j} 1_{R_{m, j}}\left(x^{(n)}\right) \frac{\int_{A} p^{n}\left(R_{m, j}\right) \mathscr{P}_{\varepsilon_{m}}(d p)}{\int_{M} p^{n}\left(R_{m, j}\right) \mathscr{P}_{\varepsilon_{m}}(d p)} \quad(A \in \mathscr{B}(M))$

Это обстоятельство имеет важное значение в связи с возможностью аппроксимировать $P\left(\tilde{p}_{\varepsilon_{m}} \in \cdot \mid \xi_{m}^{(n)}\right)(x)$ апостериорными распределениями, отвечающими смесям распределений Дирихле.

Предложенше 3. Для любого $\boldsymbol{x}$ uз $X^{\infty}$

$$
d\left(P\left(\tilde{p} \in \cdot \mid \xi_{m}^{(n)}\right)(x), P\left(\tilde{p}_{\varepsilon_{m}} \in \cdot \mid \xi_{m}^{(n)}\right)(x)\right) \leqslant \varepsilon_{m}
$$

Д ок а з те ль с в о. То же рассуждение, что было использовано при доказательстве предложения 1, показывает, что

$$
d\left(p_{\varepsilon_{m}}, p\right) \leqslant \varepsilon_{m}
$$

Далее, для любого замкнутого $A \subset M$, поскольку $p_{\varepsilon_{m}}\left(R_{m, j}\right)=p\left(R_{m, j}\right)$, имеем

и поэтому

$$
\int_{A} p^{n}\left(R_{m, j}\right) \mathscr{P}_{\varepsilon_{m}}(d p)=\int_{\left\{p: p_{\varepsilon_{m}} \in A\right\}} p^{n}\left(R_{m, j}\right) \mathscr{P}(d p)
$$

$$
\begin{aligned}
P\left(\widetilde{p}_{\varepsilon_{m}} \in A \mid \xi_{m}^{(n)}\right)(x) & =\sum_{j} \mathbf{1}_{R_{m, j}}\left(x^{(n)}\right) \frac{\int_{\left\{p_{\varepsilon_{m}} \in A\right\}} p^{n}\left(R_{m, j}\right) \mathscr{P}(d p)}{\mu^{(n)}\left(R_{m, j}\right)} \\
& \leqslant \sum_{j} \mathbf{1}_{R_{m, j}}\left(\boldsymbol{x}^{(n)}\right) \frac{\int_{\left.A^{\varepsilon_{m}}\right]} p^{n}\left(R_{m, j}\right) \mathscr{P}(d p)}{\mu^{(n)}\left(R_{m, j}\right)} \quad \text { [в силy (4.4)] } \\
& =P\left(\tilde{p} \in A^{\left.\varepsilon_{m}\right]} \mid \xi_{m}^{(n)}\right)(x) .
\end{aligned}
$$

Предложение 3 доказано.

Объединяя предложения 2 и 3 , мы получаем, что справедливо утверждение:

При выполнении условий (4.1)-(4.3)

$$
P\left(\tilde{p}_{\varepsilon_{m}} \in \cdot \mid \xi_{m}^{(n)}\right)(x) \Longrightarrow \mathscr{P}\left(\cdot ; x^{(n)}\right) \quad \text { npu } m \rightarrow+\infty, P-n . \mu .
$$

Однако вопрос о том, как выбрать $m$, не накладывая никаких дополнительных условий, чтобы расстояние Прохорова между двумя этими распределениями было меньше заданного $\varepsilon>0$, по-прежнему остается открытым. Мы вернемся к этой и смежным темам в следующем пункте. Оставшаяся часть настоящего пункта посвящена аппроксимации 
$P\left(\widetilde{p}_{\varepsilon_{m}} \in \cdot \mid \xi_{m}^{(n)}\right)(x)$ посредством апостериорных распределений, отвечающих конечным смесям в.р. Дирихле, подобно тому, как это делалось в специальном случае в разделе 5 нашей работы [13].

Пусть (как в пп. 2 и 3 ) $t_{m}$ - гомеоморфизм между $M_{\varepsilon_{m}}:=$ $\left\{\sum_{i=1}^{k_{m}} \theta_{i} \delta_{a_{m, i}}+(1-|\theta|) \delta_{a_{m, k_{m}+1}}: \theta \in T_{k_{m}}\right\}_{\text {и }} T_{k_{m}}$. Тогда для любого $x$ из $X^{\infty}$ с $\boldsymbol{x}^{(n)} \in R_{m, j}$ и для любого $A$ из $\mathscr{B}(M)$ имеем

$$
\begin{aligned}
P\left(\tilde{p}_{\varepsilon_{m}} \in A \mid \xi_{m}^{(n)}\right)(x) & =\frac{\int_{t_{m}\left(A \cap M_{\varepsilon_{m}}\right)} \theta_{1}^{n_{1}} \cdots \theta_{k_{m}}^{n_{k_{m}}}(1-|\theta|)^{n_{k_{m}+1}} d F_{\varepsilon_{m}}(\theta)}{\int_{T_{k_{m}}} \theta_{1}^{n_{1}} \cdots \theta_{k_{m}}^{n_{k_{m}}}(1-|\theta|)^{n_{k_{m}+1}} d F_{\varepsilon_{m}}(\theta)} \\
& :=Q^{\left(\varepsilon_{m}\right)}\left(t_{m}\left(A \cap M_{\varepsilon_{m}}\right) ; x^{(n)}\right)
\end{aligned}
$$

где $n_{i}=\sum_{r=1}^{n} \delta_{a_{m, i}}\left(\xi_{r, m}(x)\right)\left(i=1, \ldots, k_{m}+1\right)$. Выражение (4.5) имеет ту же структуру, что и апостериорное распределение, отвечающее ф.р. $F$, в разделе 5 нашей работы [13] с

$$
\begin{array}{cl}
F_{\varepsilon_{m}} & \text { вместо } F, \\
T_{k_{m}} & \text { вместо } T_{k}, \\
\left(a_{m, 1}, \ldots, a_{m, k_{m}+1}\right) & \text { вместо }\left(a_{1}, \ldots, a_{k+1}\right) .
\end{array}
$$

Далее, как в [13], определим

$$
\begin{aligned}
\varphi_{\lambda, \mu}^{\left(\varepsilon_{m}\right)}\left(B ; x^{(n)}\right):= & \frac{1}{\sum_{\nu} P_{F_{\varepsilon_{m}}}\left(\Delta_{\nu}\right) \gamma_{\nu}} \\
& \times \sum_{\nu} P_{F_{\epsilon_{m}}}\left(\Delta_{\nu}\right) \gamma_{\nu} \mathscr{D}^{(\lambda+n)}\left(B ; \mu_{1}+n_{1}, \ldots, \mu_{k_{m}}+n_{k_{m}}\right)
\end{aligned}
$$

$\left(B \in \mathscr{B}\left(\mathbf{R}^{k_{m}}\right)\right)$, где

$$
\gamma_{\nu}=\frac{\left(\mu_{1}\right)_{n_{1}} \cdots\left(\mu_{k}\right)_{n_{k}}(\lambda-|\mu|)_{n_{k+1}}}{(\lambda)_{n}}
$$

и

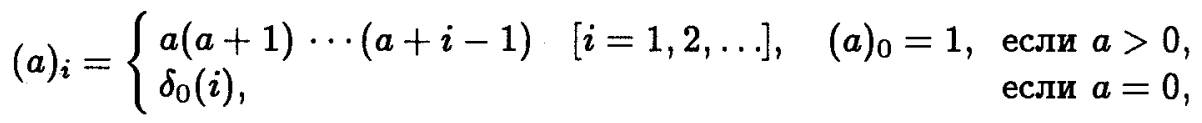

и положим

$$
\mathscr{P}_{\lambda, \mu}^{\left(\varepsilon_{m}\right)}\left(A ; x^{(n)}\right):=\varphi_{\lambda, \mu}^{\left(\varepsilon_{m}\right)}\left(t_{m}\left(A \cap M_{\varepsilon_{m}}\right) ; x^{(n)}\right) \quad(A \in \mathscr{B}(M)) .
$$

Отметим, что правая часть в последнем выражении может быть записана в виде

$$
\begin{aligned}
& \varphi_{\lambda, \mu}^{\left(\varepsilon_{m}\right)}\left(t_{m}\left(A \cap M_{\varepsilon_{m}}\right) ; x^{(n)}\right) \\
& \quad=\frac{1}{\sum_{\nu} P_{F_{c_{m}}}\left(\Delta_{\nu}\right) \gamma_{\nu}} \sum_{\nu} P_{F_{\varepsilon_{m}}}\left(\Delta_{\nu}\right) \gamma_{\nu} \mathscr{D}\left(A ; \sum_{j=1}^{k_{m}+1}\left(n_{j}+\mu_{j}\right) \delta_{a_{m, j}}\right) .
\end{aligned}
$$


Чтобы оценить точность аппроксимации $P\left(\tilde{p}_{\varepsilon_{m}} \in \cdot \mid \xi_{m}^{(n)}\right)(x)$ посредством $\mathscr{P}_{\lambda, \mu}^{\left(\varepsilon_{m}\right)}\left(\cdot ; x^{(n)}\right)$, достаточно теперь перефразировать теорему 3 из [13] и затем рассуждать так же, как при доказательстве теоремы 1.

Положив

$$
\begin{aligned}
p_{n}^{\left(\varepsilon_{m}\right)}\left(x^{(n)}\right) & =\int_{T_{k_{m}}} \theta_{1}^{n_{1}} \cdots \theta_{k_{m}}^{n_{k_{m}}}(1-|\theta|)^{n_{k_{m}+1}} d F_{\varepsilon_{m}}(\theta) \\
N_{1}^{\left(\varepsilon_{m}\right)} & =\left(2 n+k_{m}\right)\left\{\left(1+\frac{\varepsilon^{2} p_{n}^{\left(\varepsilon_{m}\right)}\left(x^{(n)}\right)}{(1+\varepsilon)(2+\varepsilon)}\right)^{1 / n\left(k_{m}+1\right)}-1\right\}^{-1}, \\
\lambda_{0}^{\left(\varepsilon_{m}\right)} & =N^{2}\left\{2 g_{k_{m}}(1-\varepsilon)^{2}\right\}^{-1} \ln \frac{2 k_{m}(1+\varepsilon)}{p_{n}^{(\varepsilon)}\left(x^{(n)}\right) \varepsilon}
\end{aligned}
$$

где $g_{k_{m}}=b_{k_{m}} \wedge\left(1-b_{k_{m}}\right)$, мы убедимся в справедливости следующего утверждения.

Лемма 1. Для любых фиксированного натурального $m, \varepsilon \in(0,1)$ и $N>N_{1}^{\left(\varepsilon_{m}\right)}$ справедливо неравенство

$$
d\left(Q^{\left(\varepsilon_{m}\right)}\left(\cdot ; x^{(n)}\right), \varphi_{\lambda, \mu}^{\left(\varepsilon_{m}\right)}\left(\cdot ; x^{(n)}\right)\right) \leqslant \varepsilon
$$

npu вcex $\lambda>\lambda_{0}^{\left(\varepsilon_{m}\right)}$.

Далее, при предположениях этой леммы для любого замкнутого $A \subset M$ имеем

$$
\begin{aligned}
\mathscr{P}_{\lambda, \mu}^{\left(\varepsilon_{m}\right)}\left(A ; x^{(n)}\right) & =\varphi_{\lambda, \mu}^{\left(\varepsilon_{m}\right)}\left(t_{m}\left(A \cap M_{\varepsilon_{m}}\right) ; x^{(n)}\right) \\
& \leqslant Q^{\left(\varepsilon_{m}\right)}\left(\left(t_{m}\left(A \cap M_{\varepsilon_{m}}\right)\right)^{\varepsilon} ; x^{(n)}\right)+\varepsilon \\
& =P\left(\tilde{p}_{\varepsilon_{m}} \in t_{m}^{-1}\left(\left(t_{m}\left(A \cap M_{\varepsilon_{m}}\right)\right)^{\varepsilon}\right) \mid \xi_{m}^{(n)}\right)(x)+\varepsilon \\
& \leqslant P\left(\tilde{p}_{\varepsilon_{m}} \in\left(A \cap M_{\varepsilon_{m}}\right)^{\left.\sqrt{k_{m} \varepsilon}\right]} \mid \xi_{m}^{(n)}\right)(x)+\varepsilon \quad[\text { в силу }(\zeta)],
\end{aligned}
$$

откуда

$$
d\left(\mathscr{P}_{\lambda, \mu}^{\left(\varepsilon_{m}\right)}\left(\cdot ; x^{(n)}\right), P\left(\widetilde{p}_{\varepsilon_{m}} \in \cdot \mid \xi_{m}^{(n)}\right)(x)\right) \leqslant \varepsilon \sqrt{k_{m}}
$$

Объединяя теперь предложения 2 и 3 с (4.6), получаем следуюший результат:

Теорема 2. Пусть $\tilde{p}_{\varepsilon_{m}}$ есть $\varepsilon_{m}$-вполне ограниченная дискретизачия $\tilde{p}$, отвечаюиая разбиению $\Pi_{m}$. Тогда для любого челого $N>N_{1}^{\left(\varepsilon_{m}\right)}$

$$
d\left(P\left(\tilde{p} \in \cdot \mid \dot{\xi}_{m}^{(n)}\right)(x), \mathscr{P}_{\lambda, \mu}^{\left(\varepsilon_{m}\right)}\left(\cdot ; x^{(n)}\right)\right) \leqslant \delta+\varepsilon_{m}
$$

всякий раз, когда $\lambda>\lambda_{0}^{\left(\varepsilon_{m}\right)}$ u $\varepsilon=\delta / \sqrt{k_{m}}$ в определениях $N_{1}^{\left(\varepsilon_{m}\right)}$ u $\lambda_{0}^{\left(\varepsilon_{m}\right)}$ Более того, если $\left(\Pi_{m}\right)_{m \geqslant 1}$ удовлетворяет условиям (4.1)-(4.3), то

$$
\lim _{m \rightarrow+\infty} d\left(\mathscr{P}_{\lambda, \mu}^{\left(\varepsilon_{m}\right)}\left(\cdot ; x^{(n)}\right), \mathscr{P}\left(\cdot ; x^{(n)}\right)\right)=0 \quad \mu^{(n)}-n . \mu .
$$


5. Aппроксимация апостериорного вероятностного распределения: дополнительные замечания. Имеются две проблемы, связанные с осуществлением процесса аппроксимации, описанного в предыдущем пункте. С одной стороны, не всегда легко явно указать эквивалентную вполне ограниченную метризацию $X$. С другой стороны, даже если такая эквивалентная метризация имеется в наличии, неясно, как найти такое $m$, чтобы расстояние $d\left(P\left(\widetilde{p} \in \cdot \mid \xi_{m}^{(n)}\right)(x), \mathscr{P}\left(\cdot ; x^{(n)}\right)\right)$ стало меньше произвольного наперед заданного числа. В связи с этим мы предлагаем в настоящем пункте иной способ дискретизаций, не использующий вполне ограниченную метризацию. При этом мы приводим также дополнительные ограничения, при выполнении которых вторая из указанных выше проблем получает свое решение. С целью упростить обозначения мы ограничиваемся случаем $n=1$.

Дискретизации, которые мы теперь рассмотрим, зависят, вообще говоря, от наблюденного $x_{1}\left(=x^{(1)}\right)$.

Пусть $\left(\varepsilon_{m}\right)_{m \geqslant 1}$ - последовательность чисел такая, что $\varepsilon_{m} \downarrow 0(m \rightarrow$ $+\infty)$, и предположим, что

$$
x_{1} \in \operatorname{support}(\mu) \subset X .
$$

Далее, выберем окрестность $A_{m}$ точки $x_{1}$ такую, что

$$
\operatorname{diam}\left(A_{m}\right) \leqslant \varepsilon_{m}
$$

Тогда $\mu\left(A_{m}\right)>0$ и существует компактное подмножество $K_{m}$ пространства $X$ такое, что

$$
x_{1} \in K_{m}, \quad \mu\left(K_{m}^{c}\right) \leqslant \varepsilon_{m}^{2} \mu\left(A_{m} \cap K_{m}\right) .
$$

Рассмотрим теперь измеримое разбиение $\left\{A_{m, 2}, \ldots, A_{m, k_{m}}\right\}$ компактного множества $K_{m} \cap A_{m}^{c}$, для которого

$$
\operatorname{diam}\left(A_{m, j}\right) \leqslant \varepsilon_{m} \quad\left(j=2, \ldots, k_{m}\right),
$$

положим

$$
K_{m}^{c}=A_{m, k_{m}+1}, \quad A_{m} \cap K_{m}=A_{m, 1}
$$

и для каждого $p$ из $M$ определим

$$
p_{\varepsilon_{m}}=\sum_{j=1}^{k_{m}+1} p\left(A_{m, j}\right) \delta_{a_{m, j}},
$$

где $a_{m, j}$ принадлежит $A_{m, j}\left(j=1, \ldots, k_{m}+1\right)$.

Дискретизация (5.1) (которую можно было бы назвать $(\varepsilon, \mu, x)$ дискретизаиией) обладает некоторыми интересными свойствами. В частности, если $C_{m}:=\left\{p \in M: p\left(A_{m, k_{m}+1}\right) \leqslant \varepsilon_{m}\right\}$, то

$$
p \in C_{m} \Rightarrow d\left(p, p_{\varepsilon_{m}}\right) \leqslant \varepsilon_{m} .
$$


Действительно, для любого замкнутого $B \subset X$ и $p$ из $C_{m}$ имеем

$$
p_{\varepsilon_{m}}(B) \leqslant \sum_{\left\{1 \leqslant i \leqslant k_{m}: a_{m, i} \in B\right\}} p\left(A_{m, i}\right)+\varepsilon_{m} \leqslant p\left(B^{\left.\varepsilon_{m}\right]}\right)+\varepsilon_{m}
$$

Следовательно, для любого замкнутого $A \subset M$ :

$$
\begin{aligned}
& P\left(\tilde{p}_{\varepsilon_{m}} \in A \mid \xi_{m}^{(1)}\right)(x)=\frac{\int_{\left\{p_{\varepsilon_{m}} \in A\right\}} p\left(A_{m, 1}\right) \mathscr{P}(d p)}{\mu\left(A_{m, 1}\right)} \\
& \quad=\frac{1}{\mu\left(A_{m, 1}\right)}\left\{\int_{\left\{p_{\varepsilon_{m}} \in A\right\} \cap C_{m}}+\int_{\left\{p_{\varepsilon_{m}} \in A\right\} \cap C_{m}^{c}}\right\} p\left(A_{m, 1}\right) \mathscr{P}(d p) \\
& \quad \leqslant P\left(\tilde{p}_{\varepsilon_{m}} \in A^{\left.\varepsilon_{m}\right]} \mid \xi_{m}^{(1)}\right)(x)+\frac{\mathscr{P}\left(C_{m}^{c}\right)}{\mu\left(A_{m, 1}\right)} \quad \text { в силу (5.2)] } \\
& \quad \leqslant P\left(\tilde{p}_{\varepsilon_{m}} \in A^{\left.\varepsilon_{m}\right]} \mid \xi_{m}^{(1)}\right)(x)+\frac{\mu\left(A_{m, k_{m}+1}\right)}{\varepsilon_{m} \mu\left(A_{m, 1}\right)} \quad \text { [по неравенству Маркова] } \\
& \quad \leqslant P\left(\tilde{p}_{\varepsilon_{m}} \in A^{\left.\varepsilon_{m}\right]} \mid \xi_{m}^{(1)}\right)(x)+\varepsilon_{m} \quad \text { [в силу (А.3)]. }
\end{aligned}
$$

Тем самым доказано следующее утверждение:

Предложение $3^{\prime}$. Если $x_{1}$ удовлетворяет (A.1), то для дискретизачии $\widetilde{p}_{\varepsilon_{m}}$ случайной в.м. $\tilde{p}$, определенной в (5.1) и обладаюоей свойствами (А.2)-(А.4), справедливо неравенство

$$
d\left(P\left(\tilde{p}_{\varepsilon_{m}} \in \cdot \mid \xi_{m}^{(1)}\right)(x), P\left(\tilde{p} \in \cdot \mid \xi_{m}^{(1)}\right)(x)\right) \leqslant \varepsilon_{m}
$$

Предложение $3^{\prime}$, по существу, совпадает с предложением 3 , но в нем нет никаких ограничений на метризацию $X$. Следовательно, первая часть теоремы 2 остается справедливой и для разбиений, использованньгх при определении $p_{\varepsilon_{m}}$ в (5.1). Пля таких разбиений, если они удовлетворяют также условиям (4.1)-(4.2) (их нетрудно построить), справедлива и вторая часть теоремы 2.

В связи с нерешенной проблемой оценки $\mathscr{P}\left(\cdot ; x_{1}\right)$ посредством $P\left(\tilde{p} \in \cdot \mid \xi_{m}^{(1)}\right)(x)$, рассмотрим специальный случай, когда выполняются следующие условия.

(В.1) Существует множество $H \in \mathscr{B}(M)$, доминированное $\sigma$-конечной мерой $\rho$ на $(X, \mathscr{B}(X))$, такое, что $\mathscr{P}(H)=1$, причем плотность (относительно $\rho$ ) каждой меры $p$ из $H$ имеет эквивалентную копию $f_{p}(x)$, для которой отображение $(x, p) \mapsto f_{p}(x)$ является $\mathscr{B}(X) \otimes(\mathscr{B}(M) \cap H)$ измеримым.

(В.2) Для любого $\varepsilon>0$ сушествует конечное измеримое разбиение $\Pi_{m}$ такое, что при $x_{1} \in A_{m, j}$

$$
I_{m, j}:=\sup _{x \in A_{m, j}} \int_{H}\left|f_{p}(x)-f_{p}\left(x_{1}\right)\right| \mathscr{P}(d p) \leqslant \frac{\varepsilon}{2} \int_{H} f_{p}\left(x_{1}\right) \mathscr{P}(d p)<+\infty .
$$

Справедливо следующее утверждение. 
Предложение 4. При выполнении (В.1)-(В.2) выполняется неравенство

$$
\sup _{B \in \mathscr{B}(M)}\left|\mathscr{P}\left(B ; x_{1}\right)-P\left(\tilde{p} \in B \mid \xi_{m}^{(1)}(x)\right)\right| \leqslant \varepsilon .
$$

Д оказат е л с т в о. Действительно, для любого множества $B$ из $\mathscr{B}(M) \cap H$ :

$$
\begin{aligned}
& \left|\frac{1}{\rho\left(A_{m, j}\right)} \int_{B} p\left(A_{m, j}\right) \mathscr{P}(d p)-\int_{B} f_{p}\left(x_{1}\right) \mathscr{P}(d p)\right| \\
& \quad=\left|\frac{1}{\rho\left(A_{m, j}\right)} \int_{A_{m, j}} \int_{B}\left\{f_{p}(x)-f_{p}\left(x_{1}\right)\right\} \mathscr{P}(d p) \rho(d x)\right| \leqslant \frac{\varepsilon}{2} \int_{H} f_{p}\left(x_{1}\right) \mathscr{P}(d p)
\end{aligned}
$$

откуда, после элементарных вычислений,

$$
\begin{aligned}
& \left|\mathscr{P}\left(B ; x_{1}\right)-P\left(\tilde{p} \in B \mid \xi_{m}^{(1)}\right)(x)\right| \\
& \quad=\left|\frac{\int_{B} f_{p}\left(x_{1}\right) \mathscr{P}(d p)}{\int_{H} f_{p}\left(x_{1}\right) \mathscr{P}(d p)}-\frac{\int_{B} p\left(A_{m, j}\right) \mathscr{P}(d p)}{\int_{H} p\left(A_{m, j}\right) \mathscr{P}(d p)}\right| \leqslant \varepsilon .
\end{aligned}
$$

Предложение 4 доказано.

Условие (В.1) выполняется почти во всех стандартных параметрических моделях. Условие (В.2) удовлетворяется, при выполнении (4.3), при всех больших $m$, если, например, семейство $\left\{f_{p}(\cdot): p \in H\right\}$ равностепенно непрерывно в $x_{1}$. Более того, из теоремы 2 следует

Предложение 5. Если условия (В.1)-(В.2) выполняются одновременно либо с (4.3), либо с (A.1)-(A.4), и если $N, \lambda$ удовлетворяют условиям, указанным в теореме 2, то

$$
d\left(\mathscr{P}_{\lambda, m}^{\left(\varepsilon_{m}\right)}\left(\cdot ; x^{(1)}\right), \mathscr{P}\left(\cdot ; x^{(1)}\right)\right) \leqslant \varepsilon+\delta+\varepsilon_{m}
$$

В нижеследующих примерах иллюстрируется предложение 5.

П р и м е р 1. Пусть $X$ есть множество всех неотрицательньх целых чисел $\mathbf{R}^{+}$. Рассмотрим множество $H \subset M$ всех абсолютно непрерывных по мере Лебега (обозначаемой в этом примере $\rho$ ) мер, имеющих гамма-плотности $a^{\gamma} e^{-a x} x^{\gamma-1} \mathbf{1}_{(0,+\infty)}(x) / \Gamma(\gamma)\left(x \in \mathbf{R}^{+}, a>0\right.$, $\gamma>0)$. Включение $H \in \mathscr{B}(M)$ легко следует, например, из того, что $H=\cup_{n \geqslant 1} H_{n}$, где $H_{n}=\left\{p \in H:(a, \gamma) \in R_{n}\right\}, R_{n}=[1 / n, n]^{2}$, и замкнутости $H_{n}$ в $M$ (последнее легко вытекает из теоремы непрерывности Леви). Предположим, что $\mathscr{P}(H)=1$. Поскольку для любого $p$ из $H$ ү $=m_{1}(p)^{2}\left\{m_{2}(p)-m_{1}(p)^{2}\right\}, a=m_{1}(p) /\left\{m_{2}(p)-m_{1}(p)^{2}\right\}$, где $m_{k}(p)=\int_{X} x^{k} p(d x)(k=1,2)$, то функция

$$
f_{p}(x):=\frac{a^{\gamma}}{\Gamma(\gamma)} e^{-a x} x^{\gamma-1} \mathbf{1}_{(0,+\infty)}(x) \quad\left(x \in \mathbf{R}^{+}, p \in H\right)
$$


является $\mathscr{B}(X) \otimes(\mathscr{B}(M) \cap H)$-измеримой. Таким образом, условие (В.1) выполнено. Далее, существует абсолютная постоянная $c$, для которой

$$
\left|f_{p}(x)-f_{p}\left(x_{1}\right)\right| \leqslant c \gamma\left(x \wedge x_{1}\right)^{-2}\left|x-x_{1}\right| \quad\left(x, x_{1}>0\right) .
$$

Предположим, что $x_{1}>0$, и рассмотрим такие разбиения $X$, что: $A_{m, 1}$ есть окрестность $x_{1}$ с $a_{1}=\inf \left\{x: x \in A_{m, 1}\right\}>0$ и $\operatorname{diam}\left(A_{m, 1}\right) \leqslant \varepsilon_{m}$, где $\varepsilon_{m} \downarrow 0$ при $m \rightarrow+\infty ; A_{m, k_{m}+1}$ есть дополнение компактного интервала $K_{m} \supset A_{m, 1}$, для которого

$$
\mu\left(A_{m, k_{m}+1}\right) \leqslant \varepsilon_{m}^{2}\left(A_{m, 1}\right)
$$

$\left\{A_{m, j}: j=2, \ldots, k_{m}\right\}$ есть разбиение множества $K_{m} \cap A_{m, 1}^{c}$ такое, что $\operatorname{diam}\left(A_{m, j}\right) \leqslant \varepsilon_{m}\left(j=2, \ldots, k_{m}\right)$.

При этом условие (В.2) оказывается выполненным, если

$$
\int_{H} \gamma \mathscr{P}(d p)<+\infty, \quad \int_{H} f_{p}\left(x_{1}\right) \mathscr{P}(d p)<+\infty
$$

и если $m$ настолько велико, что

$$
c a_{1}^{-2} \varepsilon_{m} \int_{H} \gamma \mathscr{P}(d p) \leqslant \frac{\varepsilon}{2} \int_{H} f_{p}\left(x_{1}\right) \mathscr{P}(d p),
$$

поскольку, согласно (5.3)

$$
I_{m, 1} \leqslant c a_{1}^{-2} \varepsilon_{m} \int_{H} \gamma \mathscr{P}(d p)
$$

и, по построению, $x_{1} \in A_{m, 1}$.

Наконец, предложение 5 здесь справедливо, поскольку рассматриваемые разбиения удовлетворяют условиям (А.1)-(А.4).

П р и м е $\mathrm{p} 2$. Пусть $X=\mathbf{R}^{k}$, и пусть $H$ состоит из всех невырожденньх гауссовских в.м. Плотность (соответственно, характеристическую функцию) такой в.м. со средним $\mu$ и ковариационной матрицей $V$ мы обозначим $\varphi(x ; \mu, V)$ (соответственно, $\psi(t ; \mu, V)$ ). Рассуждая как в примере 1 , нетрудно показать, что $H \in \mathscr{B}(M)$. Более того, функция $(x, p) \mapsto \varphi(x ; \mu(p), V(p)),(x, p) \in X \times H$, измерима, как это требуется B (B.1).

Далее, применяя формулу обрашения для $\psi$, можем написать при всех $x$ и $x_{1}$ из $\mathbf{R}^{k}$

$$
\begin{aligned}
\left|\varphi(x ; \mu, V)-\varphi\left(x_{1} ; \mu, V\right)\right| & \leqslant \frac{1}{(2 \pi)^{k}} \int_{\mathbf{R}^{k}}\left|\exp \left\{i\left\langle x-x_{1}, t\right\rangle\right\}-1\right||\psi(t ; \mu, V)| d t \\
& \leqslant \frac{1}{(2 \pi)^{k}}\left\|x-x_{1}\right\| h(V)
\end{aligned}
$$


где

$$
h(V)=\int\|t\| e^{-\langle V t, t\rangle / 2} d t \leqslant(2 \pi)^{k / 2}\left(\sum_{1}^{k} \lambda_{i}^{-1}\right)^{(k+1) / 2}
$$

и $\lambda_{i}$ суть собственные значения $V$.

Возьмем в качестве разбиения $X$ произведение $k$ разбиений того типа, которые использовались в примере $1: A_{m, 1}=A_{m, 1}^{(1)} \times \cdots \times A_{m, 1}^{(k)}$ c $\operatorname{diam}\left(A_{m, 1}^{(j)}\right) \leqslant \varepsilon_{m} / \sqrt{k}, x_{1} \in A_{m, 1}$ и т.д. Тогда, если $m$ настолько велико, что

$$
(2 \pi)^{-k} \varepsilon_{m} \int_{H} h(V) \mathscr{P}(d p) \leqslant \frac{\varepsilon}{2} \int_{H} \varphi\left(x_{1} ; \mu, V\right) \mathscr{P}(d p)
$$

условия (В.1)-(В.2) выполняются, равно как и (А.1)-(А.4).

П р и м е р 3 . Пусть $X$ - множество всех точечных мер на $[0, T]$, $T>0$, т.е. множество всех борелевских мер $\mu$ на $\mathscr{B}([0, T])$, для которых $\mu(A) \in \mathbf{N} \cup\{0\}$ для любого борелевского множества $A$. Наделим $X$ метрикой Леви $L$; см., например, $[7$, раздел X.13]. С этой метрикой $X$ есть польское пространство. Возьмем в качестве $H$ класс распределений $P_{\lambda}$ всех ординарных пуассоновских процессов на $[0, T]$ с интенсивностью $\lambda>0$. Рассуждение, подобное использованному в предыдущих примерах, примененное к конечномерным распределениям, вместе с хорошо известной теоремой о сходимости по распределению точечных процессов (см., например, теорему 1.21 в [10]), показывает, что $H \in \mathscr{B}(M)$. Для любого $\lambda>0$ распределение $P_{\lambda}$ абсолютно непрерывно относительно $P_{1}$ и

$$
f_{P_{\lambda}}(x):=\frac{d P_{\lambda}}{d P_{1}}(x)=\exp \left\{T(1-\lambda)-x_{T} \ln \lambda\right\} \quad(x \in X),
$$

где $x_{T}:=x([0, T])$; см. теорему 2.31 в [10]. Предположим теперь, что выполнено условие (А.1), и рассмотрим разбиение $X$, построенное в соответствии с (А.2)-(А.4) относительно метрики Леви. Тогда $x^{(1)} \in A_{m, 1}$ и

$$
\sup _{x \in A_{m, 1}}\left|f_{P_{\lambda}}(x)-f_{P_{\lambda}}\left(x_{1}\right)\right|=\sup _{x \in A_{m, 1}} \lambda^{x_{T}^{(1)}} \exp \{(1-\lambda) T\}\left|1-\lambda^{x_{T}-x_{T}^{(1)}}\right| \text {. }
$$

Более того, поскольку $\left|x_{T}-x_{T}^{(1)}\right| \leqslant L\left(x, x^{(1)}\right)$, левая часть в (5.4) равна нулю при $\varepsilon_{m}<1$. Отсюда вытекает, что условия (В.1)-(В.2) выполнены для любого разбиения пространства $X$, построенного согласно (A.2)(А.4), всякий раз, когда $\varepsilon_{m}<1$.

В заключение настояшего пункта сделаем несколько замечаний об аппроксимации предсказательного распределения $P\left(\xi_{n+j} \in A \mid \boldsymbol{\xi}^{(n)}=\boldsymbol{x}\right)$ и апостериорного в.р. функционала $\int \psi(x) \tilde{p}(d x)$ соответствующими в.р., отвечающими $\mathscr{P}_{\lambda, \mu}^{\left(\varepsilon_{m}\right)}\left(\cdot ; x^{(n)}\right)$, предполагая, что

$$
d\left(\mathscr{P}\left(\cdot ; x^{(n)}\right), \mathscr{P}_{\lambda, \mu}^{\left(\varepsilon_{m}\right)}\left(\cdot ; x^{(n)}\right)\right) \leqslant \varepsilon .
$$


Аппроксимация предсказательного вероятностного распределения. Для всякого $y$ из $\mathbf{R}$ положим

$$
\begin{aligned}
G^{A}\left(y ; x^{(n)}\right) & =\mathscr{P}\left(\{p \in M: p(A) \leqslant y\} ; x^{(n)}\right) \\
G_{\lambda, \mu}^{A}\left(y ; x^{(n)}\right) & =\mathscr{P}_{\lambda, \mu}^{\left(\varepsilon_{m}\right)}\left(\{p \in M: p(A) \leqslant y\} \cap M_{\varepsilon_{m}} ; x^{(n)}\right) \\
& =\mathscr{P}_{\lambda, \mu}^{\left(\varepsilon_{m}\right)}\left(t_{m}^{-1}\left(B_{y}\right) ; x^{(n)}\right),
\end{aligned}
$$

где $B_{y}:=\left\{\theta \in T_{k_{m}}: \sum_{i=1}^{k_{m}} \theta_{i}\left(\delta_{a_{m}, i}(A)-\delta_{a_{m}, k_{m}+1}(A)\right) \leqslant y-\delta_{m, k_{m}+1}(A)\right\}$. Тогда

$$
\begin{aligned}
& G^{A}\left(y ; x^{(n)}\right) \leqslant \mathscr{P}_{\lambda, \mu}^{\left(\varepsilon_{m}\right)}\left(\left(t_{m}^{-1}\left(B_{y}\right)\right)^{\varepsilon} ; x^{(n)}\right)+\varepsilon \quad \text { [в силу (5.5)] } \\
& =\varphi_{\lambda, \mu}^{\left(\varepsilon_{m}\right)}\left(t_{m}\left(\left(t_{m}^{-1}\left(B_{y}\right)\right)^{\varepsilon}\right) ; x^{(n)}\right)+\varepsilon \\
& \left.\leqslant \varphi_{\lambda, \mu}^{\left(\varepsilon_{m}\right)}\left(B_{y}^{2 \varepsilon} ; x^{(n)}\right)+\varepsilon \quad \text { [в силу } \zeta\right] \\
& \leqslant \varphi_{\lambda, \mu}^{\left(\varepsilon_{m}\right)}\left(B_{y+2 \varepsilon \sqrt{k_{m}}} ; x^{(n)}\right)+\varepsilon \quad\left[\text { поскольку } B_{y}^{2 \varepsilon} \subset B_{y+2 \varepsilon \sqrt{k_{m}}}\right] \\
& =G_{\lambda, \mu}^{A}\left(y+2 \varepsilon \sqrt{k_{m}} ; x^{(n)}\right)+\varepsilon \text {, }
\end{aligned}
$$

U

$$
1-G^{A}\left(y ; x^{(n)}\right) \leqslant 1-G_{\lambda, \mu}^{A}\left(y-2 \varepsilon \sqrt{k_{m}} ; x^{(n)}\right)+\varepsilon .
$$

Следовательно,

$$
\begin{aligned}
P\left(\xi_{n+j} \in A \mid \xi^{(n)}=x^{(n)}\right) & =\int_{0}^{1}\left\{1-G^{A}\left(y ; x^{(n)}\right)\right\} d y \\
& \leqslant \Phi_{\lambda, \mu}^{\left(\varepsilon_{m}\right)}\left(\xi_{n+j} \in A \mid \xi^{(n)}=x^{(n)}\right)+\varepsilon\left(1+2 \sqrt{k_{m}}\right) \\
P\left(\xi_{n+j} \in A \mid \xi^{(n)}=x^{(n)}\right) & \geqslant \Phi_{\lambda, \mu}^{\left(\varepsilon_{m}\right)}\left(\xi_{n+j} \in A \mid \xi^{(n)}=x^{(n)}\right)-\varepsilon\left(1+2 \sqrt{k_{m}}\right)
\end{aligned}
$$

Из сделанных выше замечаний вытекает следуюшее утверждение.

Предложенше 6. Если условие (5.5) выполнено для некоторого $\varepsilon>0$, mo

$$
\begin{aligned}
& \sup _{A \in \mathscr{B}(M)}\left|P\left(\xi_{n+j} \in A \mid \xi^{(n)}=x^{(n)}\right)-\Phi_{\lambda, \mu}^{\left(\varepsilon_{m}\right)}\left(\xi_{n+j} \in A \mid \xi^{(n)}=x^{(n)}\right)\right| \\
& \quad \leqslant \varepsilon\left(1+2 \sqrt{k_{m}}\right) \quad(j \in \mathbf{N})
\end{aligned}
$$

где (cp. paзden 6 в [13])

$$
\begin{aligned}
& \Phi_{\lambda, \mu}^{\left(\varepsilon_{m}\right)}\left(\xi_{n+j} \in A \mid \xi^{(n)}=x^{(n)}\right) \\
& \quad=\frac{1}{\sum_{\nu} P_{F_{\varepsilon_{m}}}\left(\Delta_{\nu}\right) \gamma_{\nu}} \sum_{\nu} P_{F_{\varepsilon_{m}}}\left(\Delta_{\nu}\right) \gamma_{\nu} \frac{\sum_{j=1}^{k_{m}+1}\left(n_{j}+\mu_{j}\right) \delta_{a_{m}, j}(A)}{\lambda+n}
\end{aligned}
$$

$u \mu_{j}, n_{j}$ u m.д. те же, что в определении $\varphi_{\lambda, \mu}^{\left(\varepsilon_{m}\right)}\left(\cdot ; \boldsymbol{x}^{(n)}\right)$ в п. 4. 
Аппроксимация апостериорного вероятностного распределения функционала $\int \psi(x) \tilde{p}(d x)$. Предположим, что $\mathscr{P}\left(\int|\psi| d \widetilde{p}<\right.$ $+\infty)=1$ и что условие (5.5) выполнено. Обозначим $H\left(\cdot ; x^{(n)}\right)$ и $H_{\lambda, \mu}^{\left(\varepsilon_{m}\right)}\left(\cdot ; x^{(n)}\right)$ ф.р. функционала $\int \psi d \tilde{p}$, отвечающие мерам $\mathscr{P}\left(\cdot ; x^{(n)}\right)$ и $\mathscr{P}_{\lambda, \mu}^{\left(\varepsilon_{m}\right)}\left(\cdot ; x^{(n)}\right)$ соответственно. Рассуждение, подобное доказательству предложения 6 , показывает, что

$$
\begin{aligned}
& H_{\lambda, \mu}^{\left(\varepsilon_{m}\right)}\left(y-\varepsilon \sqrt{k_{m}}\left\|d_{\psi}\right\|-0 ; x^{(n)}\right)-\varepsilon \leqslant H\left(y ; x^{(n)}\right) \\
& \quad \leqslant H_{\lambda, \mu}^{\left(\varepsilon_{m}\right)}\left(y+\varepsilon \sqrt{k_{m}}\left\|d_{\psi}\right\| ; x^{(n)}\right)+\varepsilon \quad(y \in \mathbf{R})
\end{aligned}
$$

где $\left\|d_{\psi}\right\|=\left(\psi\left(a_{m, 1}\right)-\psi\left(a_{m, k_{m}+1}\right), \ldots, \psi\left(a_{m, k_{m}}\right)-\psi\left(a_{m, k_{m}+1}\right)\right)$.

Как отмечалось в $[13]$, точное выражение для $H_{\lambda, \mu}^{\left(\varepsilon_{m}\right)}\left(\cdot ; x^{(n)}\right)$ легко можно получить, используя основной результат из [5], [6].

Неравенства (5.6) дают оценку сверху для расстояния Леви между $H\left(\cdot ; x^{(n)}\right)$ и $H_{\lambda, \mu}^{\left(\varepsilon_{m}\right)}\left(\cdot ; x^{(n)}\right)$.

6. Обсуждение результатов. Наши результаты могут быть использованы при выборе априорного распределения в байесовском непараметрическом статистическом анализе. Покажем, как может быть использована теорема 1.

Без существенного ограничения общности при практическом применении байесовского анализа можно считать, что класс $\mathscr{C}$ в.м. $\mu$ на $\mathscr{B}(X)$, которые являются «естественными» кандидатами, чтобы представлять в.р. каждого наблюдения, является плотныл. Из этого предположения вытекает, что накопленное знание изучаемого феномена позволяет поставить в соответствие каждому $\varepsilon>0$ компактное подмножество $K_{\varepsilon / 2}$ пространства $X$ такое, что каждый элемент класса $\mathscr{C}$ наделяет дополнение к $K_{\varepsilon / 2}$ вероятностью, не превосходящей $\varepsilon^{2} / 4$. В статистической практике в качестве $X$ выбираются (и это обычно оправдано соображениями математического удобства) некоторые стандартные выборочные пространства, включающие в себя множество всех возможных реализаций каждого наблюдения. Более того, часто предыдушее предположение выполняется с $\varepsilon=0$, и в этом случае $X$ само может быть выбрано компактным. В свете сделанных замечаний основное допущение теоремы 1 о существовании $(\varepsilon, \mu)$ - или $\varepsilon$-вполне ограниченных дискретизаций представляется вполне согласующимся с природой реальных проблем. В частности, указанное предположение влечет, что можно ограничиться рассмотрением лишь априорных распределений $\mathscr{P}$, для которых $\mu=\int_{M} p d \mathscr{P} \in \mathscr{C}$. При этом ограничении можно предложить, на основе теоремы 1 , следугщий метод для выбора априорного распределения. Первый шаг состоит в разбиении $K_{\varepsilon / 2}$ на подмножества $A_{1}, \ldots, A_{k}$, удовлетворяющие соответствующим условиям, чтобы получить $(\varepsilon / 2, \mu)$ - 
или $\varepsilon / 2$-вполне ограниченную дискретизацию. Далее, после построения разбиения $X$, можно продолжить как в разделе 6 в [13], принимая во внимание, что вместо $\left(\tilde{p}\left(a_{1}\right), \ldots, \tilde{p}\left(a_{k}\right)\right)$ мы теперь имеем $\left(\tilde{p}\left(A_{1}\right), \ldots, \widetilde{p}\left(A_{k}\right)\right)$. Более того, субъективная оценка вероятности $P_{F_{\varepsilon / 2}}\left(\Delta_{\nu}\right)$ (в обозначениях раздела 6 в [13] это $\pi_{\nu}$ ) должна быть согласована с допущением, что мера $\mu$ обязана принадлежать $\mathscr{C}$. Конечный результат этой схемы выбора есть смесь

$$
\mathscr{P}_{\lambda, \mu}^{(\epsilon / 2)}=\sum_{\nu} \pi_{\nu} \mathscr{D}\left(E ; \alpha_{\mu(\nu)}\right) \quad(E \in \mathscr{B}(M)),
$$

для которой $d\left(\mathscr{P}, \mathscr{P}_{\lambda, \mu}^{(\varepsilon)}\right) \leqslant \varepsilon$, когда $N$ и $\lambda$ удовлетворяют условиям, сформулированным в теореме 1 ; в.p. $\mu$ величины $\xi_{1}$, порожденное мерой $\mathscr{P}$, принадлежит $\mathscr{C} ; \mathscr{P}\left(\left\{p \in M:\left(p\left(A_{1}\right), \ldots, p\left(A_{k}\right)\right) \in \Delta_{\nu}\right\}\right)=\pi_{\nu}(\nu \in I)$.

Предложенная схема имеет два достоинства: с одной стороны, в ней фиксирована естественная область - семейство $\left\{\Delta_{\nu}: \nu \in I\right\}-$ для субъективной оценки априорных вероятностей, и, с другой стороны, - онаприводит к апостериорной вероятности в форме, достаточно удобной для математической обработки. Ясно, что при этом возможны практические трудности, связанные с большим числом необходимых оценок, когда число элементов в $I$ велико. Нижеследующие замечания показывают, как предложенная схема может применяться, когда имеющееся значительное число наблюдений позволяет построить $m$ частотных распределений на основе реализаций $m$ конечны $x$ непересекающихся подпоследовательностей бесконечной последовательности $\left(\xi_{n}\right)_{n \geqslant 1}$ возможных наблюдений. Отметим, что используемые «исторические» частотные распределения не обязательно должны быть эмпирическими распределениями: в действительности они могут быть «гладкими» в.р., подогнанными к наблюденным данным. В подобных ситуациях мы можем согласиться, что B.p., приписываемое $\tilde{p}$, должно быть близким к $\mathscr{P}^{*}:=\sum_{i=1}^{m} c_{i} \delta_{R_{i}}$, где $R_{1}, \ldots, R_{m}$ представляют имеюшиеся «исторические» распределения, $R_{i}$ основывается на $l_{i}$ наблюдениях и $c_{i} \simeq l_{i} / \sum_{j=1}^{m} l_{j}$ для $i=1, \ldots, m$.

Определим $K_{\varepsilon / 2}$ и $\left\{A_{1}, \ldots, A_{k}\right\}$, как это было сделано выше в настоящем пункте, и предположим, не ограничивая общности, что

$$
\theta^{(i)}\left(A_{1}, \ldots, A_{k}\right) \neq \theta^{(j)}\left(A_{1}, \ldots, A_{k}\right) \quad \text { при } i \neq j,
$$

где

$$
\theta^{(i)}\left(A_{1}, \ldots, A_{k}\right)=\left(R_{i}\left(A_{1}\right), \ldots, R_{i}\left(A_{k}\right)\right)
$$

(для этого достаточно, чтобы $R_{i} \neq R_{j}$ при $i \neq j$ и чтобы $\varepsilon$ было достаточно мало). Выберем теперь целое $N>2 k / \varepsilon$ (см. теорему 1) столь большим, чтобы каждая ячейка $\Delta_{\nu}$ содержала не более одного вектора $\theta^{(i)}$. Тогда если $\Delta_{\nu}$ содержит $\theta^{(i)}$, то $P_{F_{\varepsilon} / 2}\left(\Delta_{\nu}\right)=c_{i}$ и, по теореме 1 , 
$d\left(\mathscr{P}^{*}, \mathscr{P}_{\lambda, \mu}^{(\varepsilon / 2)}\right) \leqslant \varepsilon$, где

$$
\begin{aligned}
& \mathscr{P}_{\lambda, \mu}^{(\varepsilon / 2)}(\cdot)=\sum_{i=1}^{m} c_{i} \mathscr{D}\left(\cdot ; \alpha^{(i)}\right) ; \quad \alpha^{(i)}=\sum_{j=1}^{k+1} \mu_{j}^{(i)} \delta_{a_{j}} \\
& \mu_{j}^{(i)}= \begin{cases}0, & \text { если } R_{i}\left(A_{j}\right)=0, \\
\lambda\left\{r_{j}^{(i)}-\frac{b_{k}}{N}\right\}, & \text { если } R_{i}\left(A_{j}\right)>0,\end{cases}
\end{aligned}
$$

$i=1, \ldots, m, j=1, \ldots, k, r_{j}^{(i)}=\min \left\{n / N: n / N \geqslant R_{i}\left(A_{j}\right), n \in \mathbf{N}\right\}$ и $\lambda$ удовлетворяет (3.2) с $\varepsilon$ вместо $\varepsilon / 2 ; \mu_{k+1}^{(i)}=\lambda-\sum_{j=1}^{k} \mu_{j}^{(i)}$.

Отметим, что число параметров, значения которых нужно выбирать при определении $\mathscr{P}_{\lambda, \mu}^{(\varepsilon / 2)}$, не превосходит $m$. Более того, при подходяшем выборе $\varepsilon$ меры $\mathscr{P}_{\lambda, \mu}^{(\varepsilon / 2)}$ и $\mathscr{P}^{*}$ достаточно близки, чтобы использовать $\mathscr{P}(\varepsilon / 2)$ как удобную аппроксимацию любого априорного распределения, основанного на «объективной» информации, содержащейся в $R_{1}, \ldots, R_{m}$.

Некоторые результаты и рассуждения из п. 3 могут быть использованы для аппроксимации $\mathscr{P}^{*}$ смесями вида

$$
\mathscr{D}^{(\lambda, R)}(\cdot):=\sum_{i=1}^{m} c_{i} \mathscr{D}\left(\cdot ; \lambda R_{i}\right)
$$

Преимущество этого типа аппроксимации состоит в том, что для нее не нужно оценивать вероятности $R_{i}\left(A_{j}\right)$. Для построения такой аппроксимации отметим, что если $\mathscr{P}_{\varepsilon}^{*}$ (соответственно $\mathscr{D}_{\varepsilon}^{(\lambda, R)}$ ) есть в.р., отвечающее $\widetilde{p}_{\varepsilon}$ в предположении, что в.р., отвечающее $\widetilde{p}$, есть $\mathscr{P}^{*}$ (соответственно $\left.\mathscr{D}^{(\lambda, R)}\right)$, то, по предложению 1 ,

$$
d\left(\mathscr{P}^{*}, \mathscr{P}_{\varepsilon}^{*}\right) \leqslant \varepsilon, \quad d\left(\mathscr{D}^{(\lambda, R)}, \mathscr{D}_{\varepsilon}^{(\lambda, R)}\right) \leqslant \varepsilon .
$$

Далее, пусть $t$ означает то же, что в п. 2 , и пусть $P_{\varepsilon}^{*}=\mathscr{P}_{\varepsilon}^{*} t^{-1}, P_{\varepsilon}^{\left(\lambda_{i} R\right)}=$ $\mathscr{D}_{\varepsilon}^{(\lambda, R)} t^{-1}$, т.е. $P_{\varepsilon}^{*}=\sum_{i=1}^{m} c_{i} \delta_{R_{\varepsilon i}}, P_{\varepsilon}^{(\lambda, R)}=\sum_{i=1}^{m} c_{i} \mathscr{D}^{(\lambda)}\left(\cdot ; \lambda R_{\varepsilon i}\right)$, где $R_{\varepsilon i}=$ $\left(R_{i}\left(A_{1}\right), \ldots, R_{i}\left(A_{k}\right)\right)$ и $\left(A_{1}, \ldots, A_{k+1}\right)$ есть разбиение $X$ из определения дискретизации $\tilde{p}_{\varepsilon}$. Отметим теперь, что для любого шара $S_{\rho}\left(R_{\varepsilon i}\right)$ в $\mathbf{R}^{k}$ с центром в $R_{\varepsilon i}$ и радиусом $\rho$ и для любого случайного вектора $Y_{i}$ с в.p. $\mathscr{D}^{(\lambda)}\left(\cdot ; \lambda R_{\varepsilon i}\right)$ по неравенству Маркова

$$
\mathscr{D}^{(\lambda)}\left(\left(S_{\rho}\left(R_{\varepsilon i}\right)\right)^{c} ; \lambda R_{\varepsilon i}\right) \leqslant \frac{1}{\rho^{2}} \mathbf{E}\left(\left\|Y_{i}-R_{\varepsilon i}\right\|^{2}\right) \leqslant \frac{k}{4 \rho^{2}(\lambda+1)} .
$$

Следовательно, для любого $E$ из $\mathscr{B}\left(\mathbf{R}^{k}\right)$ имеем

$$
\begin{aligned}
P_{\varepsilon}^{(\lambda, R)}(E) & \leqslant \sum_{i=1}^{m} c_{i} \mathscr{D}^{(\lambda)}\left(E \cap S_{\rho}\left(R_{\varepsilon i}\right) ; \lambda R_{\varepsilon i}\right)+\sum_{i=1}^{m} c_{i} \mathscr{D}^{(\lambda)}\left(\left(S_{\rho}\left(R_{\varepsilon i}\right)\right)^{c} ; \lambda R_{\varepsilon i}\right) \\
& \leqslant P_{\varepsilon}^{*}\left(E^{\rho}\right)+\frac{k}{4 \rho^{2}(\lambda+1)} .
\end{aligned}
$$


Используя это неравенство и рассуждая, как при доказательстве теоремы 1 , в предположении, что

$$
\rho \leqslant \frac{\varepsilon}{\sqrt{k}}, \quad \lambda+1 \geqslant \frac{k}{4 \rho^{2} \varepsilon}
$$

получим

$$
\begin{aligned}
\mathscr{D}_{\varepsilon}^{(\lambda, R)}(H) & \leqslant \mathscr{P}_{\varepsilon}^{*}\left(\left(H \cap M_{\varepsilon}\right)^{\rho \sqrt{k}}\right)+\frac{k}{4 \rho^{2}(\lambda+1)} \quad(H \in \mathscr{B}(M)) \\
& \leqslant \mathscr{P}_{\varepsilon}^{*}\left(H^{\varepsilon}\right)+\varepsilon
\end{aligned}
$$

Таким образом, (6.3) влечет $d\left(\mathscr{P}_{\varepsilon}^{*}, \mathscr{D}_{\varepsilon}^{(\lambda, R)}\right) \leqslant \varepsilon, \mathbf{n}$, комбинируя это с (6.1), имеем

$$
d\left(\mathscr{P}^{*}, \mathscr{D}^{(\lambda, R)}\right) \leqslant 3 \varepsilon .
$$

С $\rho=\varepsilon / \sqrt{k}$ последнее неравенство справедливо при всех $\lambda>k^{2} / 4 \varepsilon^{3}-1$. Ясно, что эта нижняя грань для $\lambda$ может быть уменьшена, если в (6.2) вместо неравенства Маркова использовать более точное неравенство.

\section{СПИСОК ЛИТЕРАТУРЫ}

1. Aldous D. J. Exchangeability and related topics. - Lecture Notes in Math., 1985, v. 1117, p. 1-198.

2. Andreev A., Arjas E. A note on histogram approximation in Bayesian density estimation. - Bayesian Statistics, v. 5. Ed. by J.M. Bernardo et al. Oxford: Oxford Univ. Press, 1996, p. 487-490.

3. Биллияесли П. Сходимость вероятностных мер. М.: Наука, 1977, 352 с.

4. Billingsley P. Probability and Measure. New York: Wiley, 1995, 593 p.

5. Cifarelli D. M., Regazzini E. Distribution functions of means of a Dirichlet process. Ann. Statist., 1990, v. 18, № 1, p. 429-442 (Correction in Ann. Statist., 1994, v. 22, № 3, p. 1633-1634).

6. Cifarelli D. M., Regazzini E. Some remarks on the distribution functions of means of a Dirichlet process. - Technical Report 93.4. Milan: CNR-IAMI, 1993, 42 p.

7. Doob J. L. Measure Theory. New York: Springer-Verlag, 1994, 210 p.

8. Dudley R.M. Real Analysis and Probability. Pacific Grove, CA: Wadsworth \& Brooks/Cole Advanced Books \& Software, 1989, 436 p.

9. Ferguson T. S. A Bayesian analysis of some nonparametric problems. - Ann. Statist., 1973, v. 1, № 2, p. 209-230.

10. Karr A. F. Point Processes and their Statistical Inference. New York: Marcel Dekker, $1991,490 \mathrm{p}$.

11. Krasker W. S. A note on selecting parametric models in Bayesian inference. - Ann. Statist., 1984, v. 12, № 2, p. 751-757.

12. Прохоров Ю. В. Сходимость случайных процессов и предельные теоремы теории вероятностей. - Теория вероятн. и ее примен., 1956, т. 1, в. 2, с. 177-238.

13. Regazzini E., Sazonov V.V. Approximation of laws of multinomial parameters by mixtures of Dirichlet distributions with applications to Bayesian inference. - Acta Appl. Math., 1999, v. 58, № 1-3, p. 247-264. 